\title{
Development and Evaluation of a Stochastic Simulation of Cartilage Bone Osteogenesis
}

\author{
R.P. CHURCH and C.M. LANGTON* \\ Centre for Metabolic Bone Disease, Hull and East Yorkshire Hospitals Trust and University of Hull, Hull HU3 2RW, UK
}

(Received June 13, 2000; In final form July 11, 2000)

\begin{abstract}
Both long and irregular bones tend to form via endochondral ossification and are referred to as cartilage bones. Based upon the hypothesis that bone grows and forms as a semi-deterministic/semi-chaotic system, it should be possible to accurately model the osteogenesis of cartilage bones using a stochastic simulation. A thorough review of the literature has been undertaken enabling the cell types and tissues to be identified and a set of simulation rules to be established. The operation of the simulation has been evaluated longitudinally, reporting bone and blood vessel structures as the simulation develops to completion with fusion of the epiphyses; the simulation variability has been assessed by repeat runs using the same default conditions; and the effect of independently modifying key simulation parameters has been studied. This is thought to be the first report of a stochastic simulation of cartilage bone osteogenesis. The developed structures accurately follow the growth and form of irregular cartilage bones such as the vertebrae or calcaneus. The future of the simulation is now dependent primarily upon its potential utility in the field of bone metabolism and disease.
\end{abstract}

Keywords: osteogenesis, simulation, cartilage bone

\section{INTRODUCTION}

\section{Bone: tissue, types and formation}

Bone tissue consists of a collagen matrix onto which calcium hydroxyapatite is adsorbed. The bone tissue is organised into two structures, cortical and cancellous (or trabecular). Cortical bone is dense and compact, whereas cancellous bone consists of a complex open-celled porous framework of rod-and plate-like trabeculae interspersed with bone marrow. By con- sensus, cancellous bone has a minimum porosity of $30 \%$. Cortical bone principally serves a biomechanical support function for organs, muscles etc. whereas cancellous bone serves as a biomechanical 'shock-absorber' and a focus of high metabolic activity. There are three basic types of bone shape within the skeleton - long bones, which are approximately cylindrical in shape, with a "head" on each end (e.g. tibia); flat bones, which are not in general flat but slightly rounded in shape (e.g. cranium); and irregular bones, which are formed of shapes that do not agree

* Correspondence Address: Dr C M Langton Centre for Metabolic Bone Disease Hull Royal Infirmary Hull HU3 2RW UK Tel. 441482 675311 Fax. 441482675301 Email: c.m.langton@medschool.hull.ac.uk 
with either of the two above descriptions (e.g. vertebrae and calcanei). In a long bone, illustrated in Figure 1, cortical bone is found covering the whole outer surface but mainly at the central section (diaphysis), essentially formed as a hollow cylinder surrounding the medullary cavity. Cancellous bone is present towards the ends (metaphyses and epiphyses). Flat bones essentially consist of a layer of cancellous bone sandwiched between layers of cortical bone. Irregular bones consist predominantly of cancellous bone (up to 95\%) with a thin cortical shell. A thin layer of periosteum covers the surface of all bones, consisting of resting osteogenic cells.

Bones are developed and formed in two ways intermembranous ossification, where the bone forms directly from the mesenchyme via differentiation into osteoblasts, and endochondral ossification, where a cartilage model is produced initially, which is then calcified by hypertrophication of chondrocytes and ossified. As a general rule, flat bones tend to form via intermembranous ossification, and are therefore commonly referred to as membrane bones, whereas long and irregular bones tend to form via endochondral ossification and are referred to as cartilage bones. This paper describes the stochastic simulation of bone development and formation (osteogenesis) of cartilage bones.

\section{DETERMINISTIC AND CHAOTIC MECHANISMS OF CANCELLOUS BONE OSTEOGENESIS}

If one considers the structure of cancellous bone, illustrated in Figure 2, although there appears to be an underlying deterministic pattern as predicted by Wolff's law ${ }^{6}$, where the general trajectory of the trabeculae follow the principal lines of stress experienced by the bone, there is still a significant degree of variability and randomness. This is supported by recent work demonstrating that there is a strong relationship between mechanical and fractal properties of cancellous bone $e^{1,5}$ and evidence that realistic structures simulating those of cancellous bone may be created using fractal modelling ${ }^{4}$. This leads us to propose

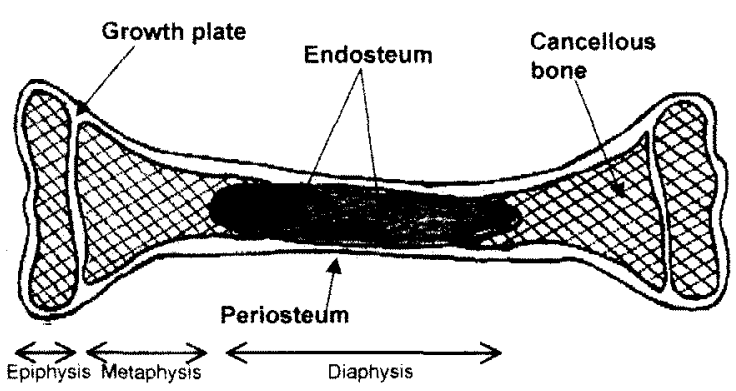

FIGURE 1 Diagramatic representation of the bony tissues associated with a typical long bone. Note that irregular cartilage bones such as the vertebra and calcaneus do not have a diaphysis section

that cancellous bone grows and forms as a semi-deterministic/semi-chaotic system and hence could be accurately modelled using a stochastic simulation.

Complementary to this, we have recently described a dynamic stochastic simulation of cancellous bone resorption ${ }^{2}$ based upon the concept of the basic multicellular unit (BMU), where net resorption $(-\triangle \mathrm{B} . \mathrm{BMU})$ was considered at bone/marrow surfaces. The simulation was applied to a simple 2D matrix lattice structure representing vertebral cancellous bone, consisting of square bone or marrow elements of approximately $20 \mu \mathrm{m}$ in dimension. The simulation considered the probability that any surface element would be activated into a BMU, and if activated, the length of the resorption cavity. The mechanical stiffness of each resultant structure was calculated by finite element analysis. As an example of its potential clinical utility, the simulation has been used to investigate the recovery in cancellous bone stiffness resulting from a stochastic simulation of anabolic treatment following varying degrees of resorption ${ }^{3}$. The simulation demonstrated that during osteoporosis, the stiffness of cancellous bone decreases at twice the rate that bone density is lost. When the original density was regained due to anabolic treatment, the stiffness of the structure was lower than the original stiffness, the deviation being linearly proportional to the degree of initial resorption prior to anabolic simulation. Hence, a significant increase in cancellous bone density is required in order to regain the original stiffness. 


\section{a) Iliac Crest}

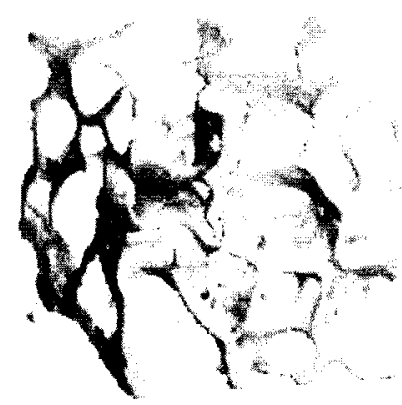

b) Femoral Head

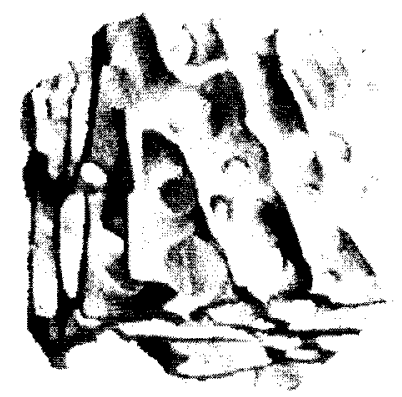

\section{c) Lumbar Spine}

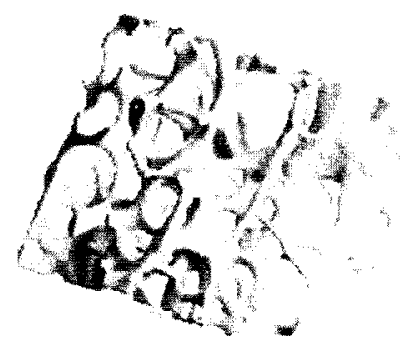

FIGURE 2 3D micro-CT reconstructions of human cancellous bone from a) iliac crest, b)femoral head, and c) lumbar spine. The complex trabecular patterns follow the principle line of stress endured yet maintain a high degree of variability and randomness. (Images provided by Professor P. Ruegsegger, University of Zurich)

\section{Formation of cartilage bones}

Cartilage consists of a dense network of collagenous fibres (with some elastic fibres) surrounded by a ground substance in the form of a gel. Cartilage bones form first by the formation of cartilage, then by the ossification of the formed cartilage. A flow-chart describing cartilage bone osteogenesis is shown in Figure 3, to which the following description of the various development processes should be referred.

1. Initially, mesenchymal (undifferentiated 'building block' cells) cells condense into the shape of a particular "model" of the bone. These cells are largely undifferentiated and have oval nuclei and fused cytoplasm.

2. The condensed mesenchymal cells then differentiate into chondroblasts (cartilage forming cells) and the intercellular matrix is laid down - this forms a stiff gel that accounts for the stiffness and flexibility of cartilage. When the chondroblasts have laid down matrix surrounding themselves, they differentiate into chondrocytes (cartilage cells).

3. The cells on the outside of the model form the perichondrium, which has two layers. The outer layer differentiates into fibroblasts, which produce collagen fibres. The inner layer remains as undifferentiated mesenchymal cells. The perichondrium forms a transition from the cartilage tissue to the tissue surrounding it.

4. Cartilage grows in two directions, along the length and breadth of the model. Lengthways growth is due to interstitial growth (from within) by mitosis of the cells close to the ends of the model - there are closely defined growth areas where this takes place. Thickening is due to appositional growth (on the surface) by mitosis of the mesenchymal cells on the inside edge of the perichondrium, some of which then differentiate into chondrocytes.

5. As the model grows from the centre outwards, the oldest chondrocytes are those at the centre of the model. These mature chondrocytes hypertrophy, enlarging the spaces in the ground material that they occupy, and then begin to produce alkaline phosphatase. This causes the cartilage to calcify (crystals of calcium phosphate build up on the organic matrix). However, as the minerals and nutrients for the chondrocytes cannot diffuse through the calcified cartilage in the same way 


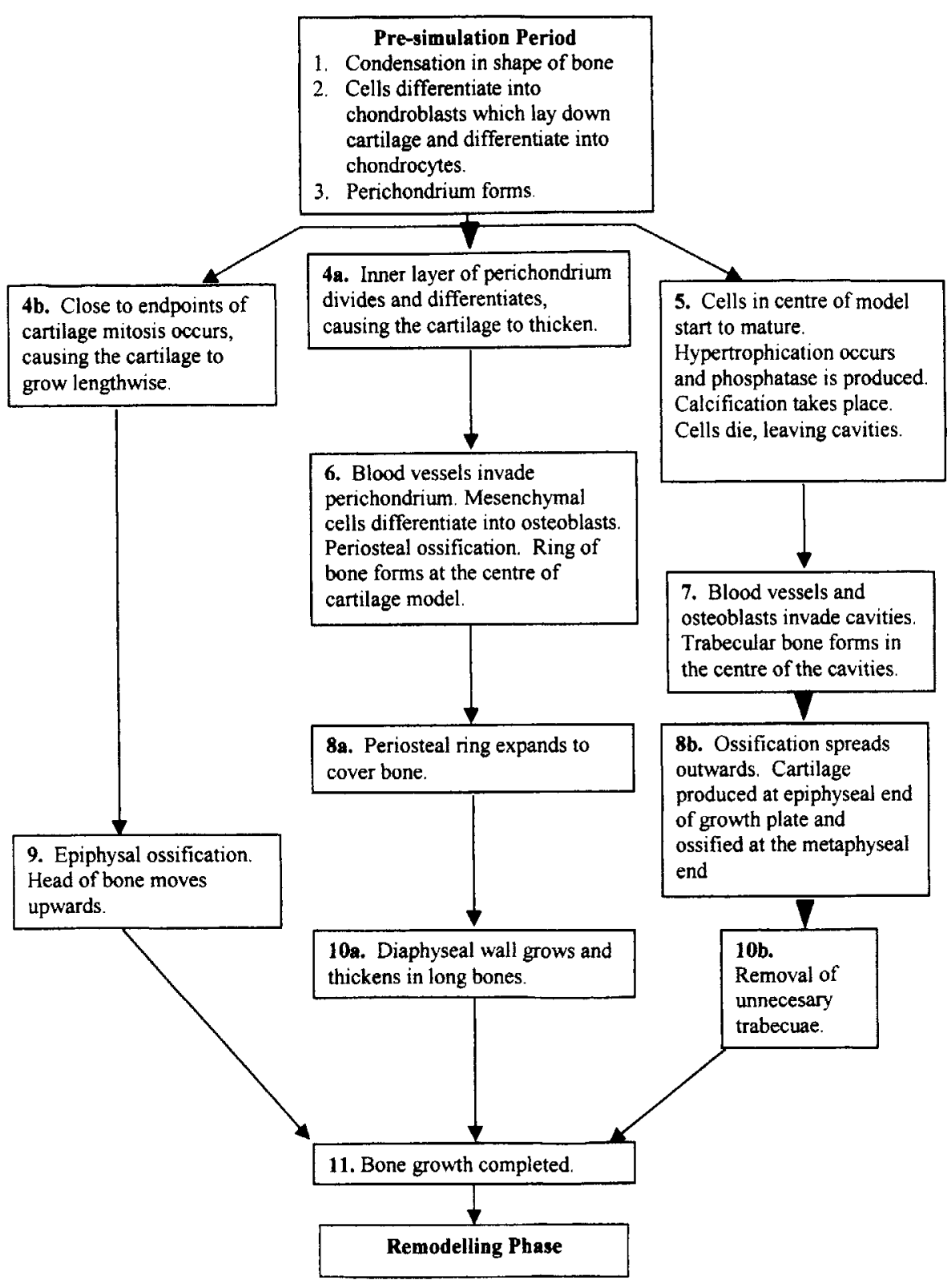

FIGURE 3 Flow chart defining the various stages of cartilage bone osteogenesis. Note that the current simulation commences at stage 4 and that there are three concurrent pathways

that they can through ordinary cartilage, the chondrocytes die. This then leaves large cavities with calcified cartilage surrounding them, which slowly decays in the absence of the chondrocyte system to maintain it.
6. As the model enlarges, blood vessels invade the perichondrium at the centre of the model. At this point, the inner layer of mesenchymal cells then differentiates into osteoblasts and osteogenic cells. These cause a layer of bone to be laid down on the 


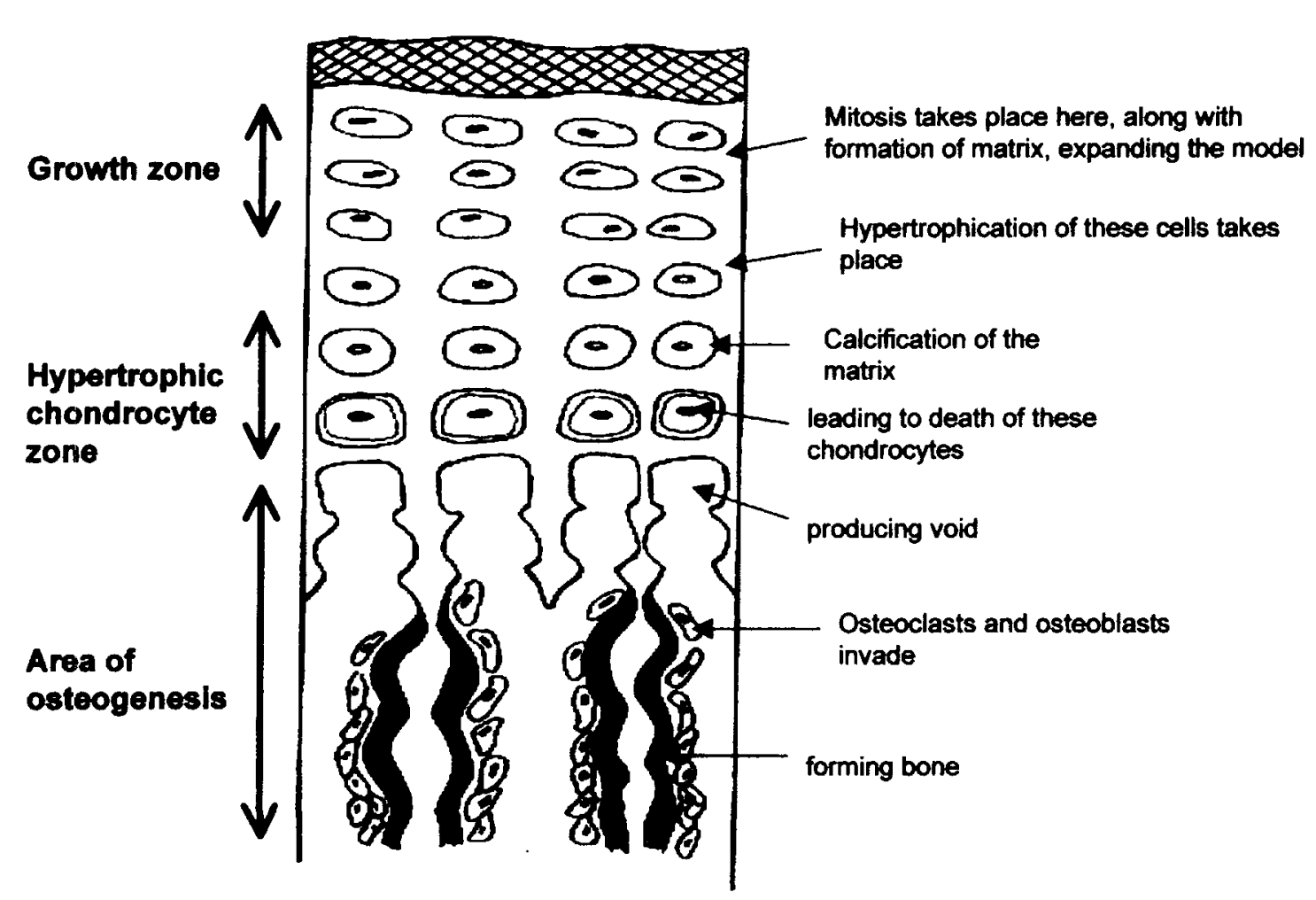

FIGURE 4 Diagramatic representation of cartilage bone formation (adapted from Ham and Leeson, Histology, 1961)

inside of the perichondrium, leading to a ring of bone around the centre of the model. This means that the membrane that was the perichondrium is now covering bone, and therefore it is referred to as the periosteum. It still retains its basic two-layer structure.

7. The cavities left by the death of the chondrocytes are invaded by blood vessels, osteoblasts and osteoclasts from the periosteum. The osteoclasts remove some of the calcified cartilage and the osteoblasts lay down a layer of bone on top of the cartilage that remains. This leads to the characteristic open framework of cancellous bone.

8. Periosteal ossification keeps the end of the periosteal bone level with the hypertrophied cartilage. Blood vessels running longitudinally along the edges of the model are surrounded with bone and Haversian systems are formed. The older remain- ing cartilage cells, those closest to the calcified cartilage already formed and converted into bone, undergo the process of hypertrophication, calcification and death. The cavities remaining are then invaded by osteoblasts from closer to the centre of the bone, causing ossification. Thus the boney part of the model expands.

9. Conversion of cartilage to bone starts at two other secondary centres of ossification in the epiphysis, as shown in Figure 4. A growth plate of cartilage forms between the cancellous bone of the metaphysis and that of the epiphysis, as shown in Figure 1. The growth plate can be split into four sections - resting cartilage adjacent to the epiphysis; proliferating cartilage where the cells are undergoing mitosis, enlarging the model; maturing cartilage, where the cartilage is ageing and becoming hypertrophied; and calcified cartilage, 
where the cartilage has become calcified due to the action of phosphatase enzyme. Thus at the epiphyseal end of the growth plate, new cartilage is being produced and at the metaphyseal end, it is being converted into bone. The bone grows in width by deposition on the outside and resorption on the inside. As the epiphysis grows, the "head" of the bone moves further along the structure, again by absorption and deposition.

10. Deposition continues on the outer surface of the pereosteal bone. Resorption also takes place on the inner wall due to osteoclastic activity, but at a slower rate, which leads to a thickening of the periosteal bone and an increase in the bone radius overall. As the periosteal bone expands, many of the central trabeculae loose their mechanical function supporting the bone and are removed by osteoclastic activity as they are no longer necessary.

11. When growth of the bone is complete, the growth plate is completely ossified and the metaphysis and epiphysis fuse. The only cartilage that now remains is that forming the joint at the end of the bone.

\section{METHOD}

The stochastic simulation of osteogenesis was implemented in MATLAB (version 5.0, Mathworks Inc., Natick, US).

\section{Fundamental basis of the stochastic simulation}

The chronological starting point for the simulation was chosen to be the initial cartilage phase of a generic bone. The model is based around a 2-dimensional matrix where square elements are assigned values that represent the substance or cell present within a particular element. A small piece of cartilage is initially present in a mostly empty matrix and grows to fill it. The size of the various components within the model is crucial. Thus it is necessary to have a list of the components involved so that their elemental sizes may be defined. Table I describes the various compo- nents incorporated within the simulation along with their corresponding size.

The data indicates that an elemental spatial resolution of $20 \mu \mathrm{m}$ would provide an acceptable compromise between physiological accuracy with memory requirements and simulation running time, noting that a sample of cartilage $1 \mathrm{~cm}$ by $5 \mathrm{~cm}$ would contain 1.25 million elements. It is acknowledged that the cells involved are not square in reality, but this deviation will not detract from the validity of the simulation. The elemental size attributed to each cell is indicated in Table I. The operation of the model is based upon the contents of each element. Each component within the model is therefore allocated a specific code, indicated in Table I. The code for an empty space is 0 , cell codes lie between 1 and 9, and tissues (bone, cartilage, etc) codes are between 10 and 13. The codes for the chondrocytes, osteoblasts and osteoclasts vary across a non-integer range. This is because the codes include the reciprocal of the age of the cell (in cycles). Using this arrangement, a range of ages that goes as far upwards as necessary may be stored without having to create another array or use a large range of data to store the ages. For a chondrocyte, the value is simply the reciprocal of the age; for the osteoblasts and osteoclasts, the reciprocal of the age is added to the starting value.

A second matrix of the same dimensions is used to contain the structure of the blood vessels, using a similar coding system where void is zero; a blood vessel developing up, down, left or right is coded 1 to 4 respectively; a stable blood vessel being coded 5 . Thus it is possible to have, for example, bone and a blood vessel in the same place. This is necessary as the blood vessels and capillaries grow through the tissue to supply blood to the different cells present.

\section{Cartilage implementation}

Cartilage consists of cells arranged in strips parallel to the length of the model. The cells are closer together, parallel to the length of the bone than they are in the two directions perpendicular to it. The space between the cells is filled with an intercellular matrix of collagen and ground substance. 
TABLE I Description of simulation components, along with their real size, elemental dimension within the simulation matrix, along with simulation coding and image colour

\begin{tabular}{ccccc}
\hline Component & Size & Elements & Code & Colour \\
\hline Chondrocyte & Cells & & & \\
Hypertrophic chondrocyte & $10 \mu \mathrm{m}-20 \mu \mathrm{m}$ & $1 \times 1$ & $0<\mathrm{n} \leq 1$ & Blue \\
Growth zone chondrocyte & $\approx 60 \mu \mathrm{m}-100 \mu \mathrm{m}$ & $3-5 \times 3-5$ & 2 & Dark Blue \\
Osteoblast & $\approx 60 \mu \mathrm{m}-100 \mu \mathrm{m}$ & $1 \times 1$ & 3 & Light Blue \\
Osteoclast & $20 \mu \mathrm{m}-30 \mu \mathrm{m}$ & $1 \times 1$ & $3<\mathrm{n} \leq 4$ & Green \\
Osteocyte & $10 \mu \mathrm{m}-100 \mu \mathrm{m}$ & $1-2 \times 1-4$ & $4<\mathrm{n} \leq 5$ & Red \\
Mesenchymal cell & $10 \mu \mathrm{m}-20 \mu \mathrm{m}$ & $1 \times 1$ & 6 & Dark Green \\
Tissues & $\approx 10 \mu \mathrm{m}-20 \mu \mathrm{m}$ & $1 \times 1$ & 7 & Yellow \\
Perichondrium & & & & Bright Pink \\
Periosteum & & & 9 & Purple \\
Bone & & & 10 & White \\
Cartilage & & & 11 & Grey \\
Calcified cartilage & & & 12 & Light Grey \\
Marrow & & & Pale Pink \\
External void & & & Matrix & Red Dark Red
\end{tabular}

Initially, the chondrocytes (cartilage cells) are of the order of $20 \mu \mathrm{m}$ in diameter. In the simulation, they are represented as 1 -unit blocks. Note that with this arrangement, hypertrophied cells are able to come into contact with each other, but not overlap.

Cartilage growth takes place by cell division, there being a finite but small probability that any chondrocyte will undergo mitosis in any given period, with a much higher probability that the chondrocytes in the growth zones will undergo mitosis. The other cells around the dividing cell "move up" by a fixed amount to compensate, thus enlarging the tissue. The cells move in the direction of the nearest longitudinal end of the bone. It is important to note that the growth zones move towards the end of the model as this process takes place, not staying in the same place or moving inwards towards the centre.

When the mesenchymal cells on the inside of the perichondrium divide, some of them differentiate into chondrocytes and some of them form cartilage, depending on whether there are chondrocytes in the vicinity. For the purpose of the simulation, elements centred on the cell and within a $7 \times 7$ area are considered. Older chondrocytes are more likely to mature and hypertrophy, simulated by a function where the probability of maturation is proportional to the age squared plus an exponential component to "terminate" the life of the chondrocytes at a certain point. Hypertrophied chondrocytes cause the surrounding tissue to be calcified by secreting phosphatase. This is also modelled as a stochastic process - any element of cartilage within a given distance of a hypertrophied chondrocyte will have a fixed probability of being calcified. When a hypertrophied chondrocyte no longer has access to cartilage (through calcification of the cartilage surrounding it) it dies, leaving a void.

When an element has been evaluated within a particular cycle, it is labelled as such, including elements that have grown during that cycle. This prevents some elements being evaluated more than once or not at all. For each cycle, the program scans through the elements until there are no unevaluated elements left. 
TABLE II Simulation rule set identifying and detailing the separate procedures undertaken for cell type and tissue incorporated within the simulation

\begin{tabular}{|c|c|c|}
\hline Cell Type & Procedure & Action \\
\hline \multirow[t]{3}{*}{ Chondrocyte } & Maturation & $\begin{array}{l}\text { Random probability of maturation and hypertrophication related to age. If hypertophied, expand randomly } \\
\text { by } 5 \times 5 \text { or } 3 \times 3 \text { elements. }\end{array}$ \\
\hline & Reproduction & $\begin{array}{l}\text { Random probability of mitosis, cell moving towards nearest longitudinal bone end, new chondrocyte } \\
\text { formed. }\end{array}$ \\
\hline & Age & Incremental age of chondrocyte. \\
\hline \multirow[t]{2}{*}{ Hypertrophic Chondrocyte } & Calcification & Random probability of surrounding cartilage becoming calcified cartilage. \\
\hline & Death due to Inability to Absorb Nutrients & If none of direct neighbours cartilage, cell dies and is replaced by marrow. \\
\hline Growth Zone Chondrocyte & Reproduction & $\begin{array}{l}\text { Random probability of mitosis, cell moving towards nearest longitudinal bone end, new chondrocyte } \\
\text { formed. }\end{array}$ \\
\hline \multirow[t]{4}{*}{ Osteoblast } & Ossification & $\begin{array}{l}\text { If any neighbours }(7 \times 7 \text { area) are calcified cartilage, random probability for bone formation. If any neigh- } \\
\text { bours are void or cartilage, similar process is undertaken incorporating a separate random probability. }\end{array}$ \\
\hline & Movement & Random probability for direction and activity. \\
\hline & Differentiation & If all eight neighbours are bone or calcified tissue, differentiate into osteocyte. \\
\hline & Death due to Age & $\begin{array}{l}\text { Incremental age: if beyond defined age, cell dies if less than six neighbours bone or calcified cartilage else } \\
\text { differentiates into osteocyte. }\end{array}$ \\
\hline \multirow[t]{3}{*}{ Osteoclast } & Resorption & If any neighbours are bone or calcified cartilage, random probability of forming resorption pit. \\
\hline & Movement & Random probability for direction and activity. \\
\hline & Death due to Age & Incremental age: if beyond defined age, replaced by osteoblast. \\
\hline \multirow[t]{3}{*}{ Mesenchymal Cell } & Perichondrium Formation & Convert void neighbours into perichondrium. \\
\hline & Surrounded Differentiation & $\begin{array}{l}\text { If directly ( } 3 \times 3 \text { ) surrounded by solid tissue (bone/cartilage/calcified cartilage), look at } 7 \times 7 \text { area. If no } \\
\text { chondrocytes present, change element into chondrocyte; if chondrocyte within } 7 \times 7 \text { area, change element } \\
\text { into cartilage. If no cartilage within } 3 \times 3 \text { area, differentiate into osteoblast. }\end{array}$ \\
\hline & Growth & $\begin{array}{l}\text { If no blood vessels in vicinity, random probability of growth leaving cartilage. If blood vessels present, } \\
\text { random probability of growth creating osteoblast. }\end{array}$ \\
\hline \multirow[t]{3}{*}{ Perichondrium } & Surface Check & If no void in vicinity, convert into cartilage. \\
\hline & Cartilage Check & If cartilage in vicinity, convert into mesenchymal cell. \\
\hline & Bone Check & If bone in vicinity, convert into periosteum. \\
\hline Periosteum & Surface Check & If no void in vicinity, convert into bone. \\
\hline Bone & Surface Conversion & If void in vicinity, convert into periosteum. \\
\hline Cartilage & Surface Conversion & If void in vicinity, convert into mesenchymal cell. \\
\hline Calcified Cartilage & Random Decay & If no chondrocyte within vicinity and at least 2 void elements, random allocation of conversion into void. \\
\hline Marrow & None & None \\
\hline
\end{tabular}


TABLE III Simulation rule set for the development of blood vessel, identifying and detailing the procedures for each tissue

\begin{tabular}{|c|c|c|}
\hline Component & Procedure & Action \\
\hline Void & None & None \\
\hline \multirow[t]{4}{*}{ Active Blood Vessel } & Growth & Random degree of growth. \\
\hline & Branching & Random probability of branching. \\
\hline & Osteogenic Cell Production & $\begin{array}{l}\text { If calcified tissue and up to two osteogenic cells in vicinity, random probability for } \\
\text { cell creation, if so, random probability of osteoblast or osteoclast created. }\end{array}$ \\
\hline & Age Stability & If beyond defined age, convert to stable blood vessel. \\
\hline Stable Blood Vessel & Osteogenic cell production & $\begin{array}{l}\text { If calcified tissue and up to two osteogenic cells in vicinity, random probability for } \\
\text { cell creation, if so, random probability of osteoblast or osteoclast created. }\end{array}$ \\
\hline
\end{tabular}

TABLE IV The parameters incorporated within the stochastic simulation of osteogenesis, indicating their program label and default value

\begin{tabular}{lcc}
\hline \multicolumn{1}{c}{ Parameter } & Name & Default Value \\
\hline End of growth age & growth_maxape & 50 \\
Cycles between the end of growth and program termination & growthend & 5 \\
Chondrocyte maturing constant & cc mature & 0.0002 \\
Hypertrophied chondrocyte size constant & hcc_size & 0.5 \\
Chondrocyte growth constant & cc_grow & 0.0005 \\
Calcification constant & ccar_form & 0.1 \\
Growth zone chondrocyte growth constant & gzcc_grow & 0.3 \\
Osteoblast deposition constant on calcified cartilage & ob_depositl & 0.2 \\
Osteoblast deposition constant elsewhere & ob_deposit2 & 0.0005 \\
Maximum permitted osteoblast age & ob_maxage & 5 \\
Osteoclast resorption constant & ocl_resorb & 0.5 \\
Maximum permitted osteoclast age & ocl_maxape & 5 \\
Mesenchymal cell growth constant & ms_grow & 0.1 \\
Mesenchymal cell growth constant near blood vessels & ams_grow & 0.75 \\
Calcified cartilage decay constant & ccar_decay & 0.001 \\
Vascular growth factor & bv_grow & 1 \\
Maximum blood vessel growth distance & bv_grow_distance & 4 \\
Vascular branching factor & bv_branch & 0.05 \\
Osteogenic cell production factor & bv_spawn & 0.07 \\
Osteogenic cell decision factor & bv_choose & 0.9 \\
Maximum active blood vessel age & bv_maxage & 5 \\
\hline
\end{tabular}

TABLE V Comparison of simulation stages with chronological age and typical simulation age (number of cycles)

\begin{tabular}{rcc}
\hline Stage (Figure 3) & Chronological Age & Simulation Age (cycles) \\
\hline 1 & 26 days (from ovulation) & Pre-model \\
2 & 35 days & Pre-model \\
3 & 35 days & Pre-model \\
4 & 39 days onwards & 1 onwards
\end{tabular}




\begin{tabular}{ccc}
\hline Stage (Figure 3) & Chronological Age & Simulation Age (cycles) \\
\hline 5 & 43 days onwards & 10 onwards \\
6 & 45 days & 5 onwards \\
7 & 47 days & 15 onwards \\
8 & 12 th week & 30 \\
9 & 10 th week onwards & 35 \\
10 & 12 th week onwards & not applicable \\
11 & $18-20$ years after birth. & At growth_maxage (typically 50) \\
\hline
\end{tabular}

\section{Implementation of growth period}

During each cycle the simulation performs a series of procedures based upon the defined 'Rule Set' of the osteogenesis simulation, defined in Table II.

A similar process is undertaken for the blood vessel matrix, indicated in Table III.

Once all elements have been assessed and appropriate actions implemented, the osteogenesis timer is incremented by one cycle. If the timer is equal to a pre-defined value, all growth zone chondrocytes are converted into normal chondrocytes and all growth constants reset to zero. There is a further period when remaining chondrocytes (normal or hypertrophied) mature and die, and the calcified cartilage that they form is ossified, before the simulation would move into a bone remodelling phase.

The parameters incorporated within the simulation are identified in Table IV. Other than parameters relating to ages (which are measured in cycles), they are in the form of probabilities - i.e. the chondrocyte growth constant is the probability that a chondrocyte will grow within any one cycle.

\section{RESULTS}

It is possible to broadly relate the number of simulation cycles to both the stages of osteogenesis (Figure 3) and the chronological age of the developing child, shown in Table V. It should be noted however that these can only be approximate relationships since different skeletal cartilage bones will develop at different rates, over varying timescales, in different foetuses.

The simulation was run with default parameter values and screenshots taken at various stages of osteogenesis (refering to the flowchart, Figure 3). At zero simulation age (zero cycles), shown in Figure 5a, the cartilage bone is at the beginning of stage 4 (Figure 3), the first three stages having been already completed. This is the initial cartilaginous model from which the growth begins. The chondrocytes are arranged in rows parallel to the length of the model. Two growth zone chondrocyte plates are along the vertical edges of the model, these are the points from which the majority of the growth of the bone will take place. Around the outside of the model is a layer of mesenchymal cells enveloped by a layer of perichondrium. The origins of blood vessel structure, shown in Figure $5 \mathrm{~b}$, are two long vessels along the top and bottom of the matrix, and two points nearer the centre. This provides a set of blood vessels that grow to produce a realistic process, in practice these would surround the cartilaginous model.

At a simulation age of approximately 9 cycles, shown in Figure 6a, the simulation has moved to the beginning of stages 5 and 6 where the hypertrophication of cartilage cells has just begun. Near the centre and towards the lower left of the model, patches of marrow can be seen where hypertrophied chondrocytes, having calcified the cartilage around themselves, have died. This leaves the characteristic patch of marrow (pale pink) with calcified cartilage (light grey) surrounding it. At the top centre, two newly calcified cartilage cells can be seen. The vascular invasion of the perichondrium can also be seen occurring 


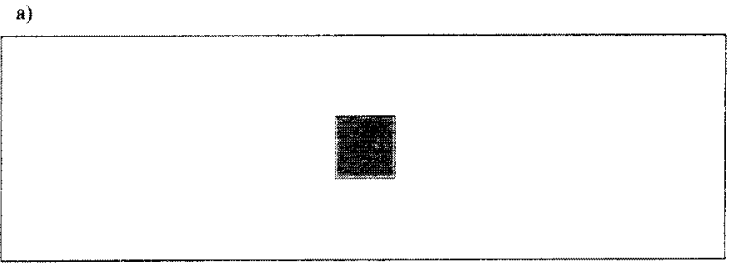

b)

FIGURE 5 The initial (zero cycles) simulation structures from which the cartilage bone grows. In the cell and tissue model (a), the chondrocytes are arranged in rows parallel to the length of the model. The growth zone chondrocyte plates are along the two vertical edges of the model. A layer of mesenchymal cells are positioned around the outside of the model, enveloped by a layer of perichondrium. The origins of the blood vessel structure (b) are two long vessels along the top and bottom of the matrix, and two points nearer the centre. The box indicates the area of the simulation matrix, being identical in size for the cell / tissue and blood vessel models. In practice, the simulation program super-imposes the two matrices

at the top and bottom of the model. At the bottom of the model, the osteoblasts (green) are clearly visible, whereas at the top, some ossification can be seen to have taken place with flecks of bone (white) being visible. Growth that has taken place at this point is quite ragged, hence the ends of the model are quite uneven. The blood vessels at this stage have started proliferating towards the centre of the model, shown in Figure 6b, noting that at this point, the majority of the blood vessels are active (light red).

Stage 5 is well illustrated in Figure $7 \mathrm{a}$, at a simulation age of 16 cycles. Here, the hypertrophication of cartilage cells, the formation of calcified cartilage and the ossification of this cartilage are all taking place clearly at the top of the model, and to a lesser extent at the bottom. Again at the bottom, the formation of periosteal bone can be seen more clearly. At the centre left, the beginning of the hypertrophication of the cartilage cells that will make up the epiphysis can be seen. Growth of the model is still very uneven - this a)
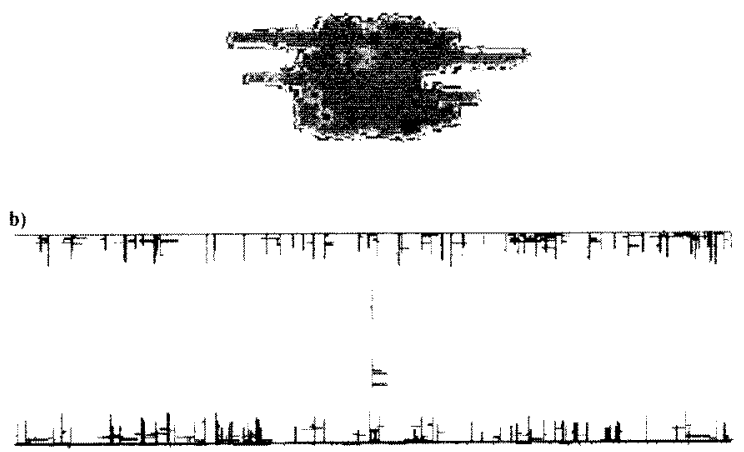

FIGURE 6 Corresponding cell \& tissue model (a) after nine cycles where hypertrophication of cartilage cells has just begun; the blood vessel structure (b) proliferating towards the centre of the model

is primarily due to the random probabilities incorporated within the simulation. The blood vessels growing vertically have met in the centre of the model, illustrated in Figure 7b. Further, the points where they coincide with the surface of the model are indicated by the presence of osteoblasts (green) at those points, shown in Figure $7 \mathrm{a}$.

After 23 cycles, stages 6 to 8 are in operation, shown in Figure 8a. Here, the ossification of the calcified cartilage can be seen even more clearly, with the bone produced forming a band down the centre of the model. Hypertrophication of the chondrocytes has clearly spread towards the edges of the model, but has in general not caught up with the growth zone chondrocytes, most of which are still visible. Periosteal ossification is visible around the outside of the bone, with most of the mesenchymal cells on the top and bottom of the model having differentiated into osteoblasts. In some places, the periosteal bone is also visible. Note that the growth of the model at the extremities is still in evidence as the model continues to expand, remaining quite uneven, although less marked than earlier. The blood vessels originating at the bottom of the matrix, shown in Figure 8b, can now be seen to be reaching the edge of the cartilaginous model causing the differentiation of mesenchymal cells, shown in Figure 8a. 
a)

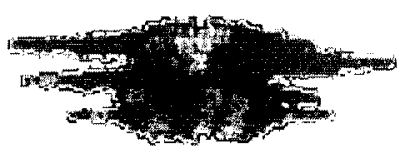

b)

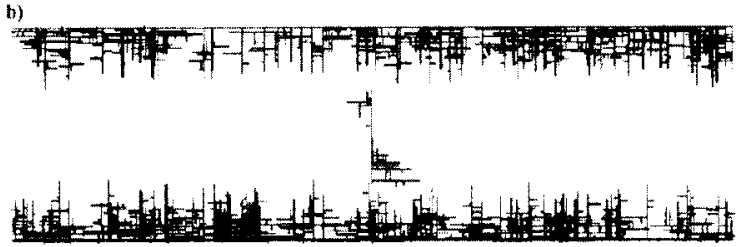

FIGURE 7 After 16 cycles, in the cell and tissue model (a), hypertrophication of cartilage cells, the formation of calcified cartilage and the ossification of this cartilage are all taking place. The blood vessels (b) have met in the centre of the model, the points where they coincide with the surface of the model indicated by the presence of osteoblasts

At a simulation age of approximately 30 cycles, stages 7 and 8 may be observed, shown in Figure 9a. At this point, the periosteal bone can be seen, especially at the top of the model. A large quantity of osteoblasts have been generated due to the vascular invasion of the model, and these have caused the ring of bone to be laid down. Most of these osteoblasts, formed by the differentiation of mesenchymal cells, have now differentiated or died, but those along the sides of the model are still easily visible. Further osteoblastic activity ossifying the calcified cartilage inside the model can also be seen, along with a small number of osteoclasts. It is possible to see the beginnings of a trabecular structure forming within the model as the calcified cartilage is ossified. Figure $9 \mathrm{~b}$ indicates vascular invasion of the model, resulting in periosteal ossification. Some of the blood vessels have reached the inside of the model causing the ossification of trabeculae away from the centre of the model.

Stages 7 and 9 are in operation after 34 simulation cycles, the formation of trabeculae being quite pronounced, shown in Figure 10a. Hypertrophication of chondrocytes and calcification of cartilage is still taking place closer to the ends of the bone, and the region a)
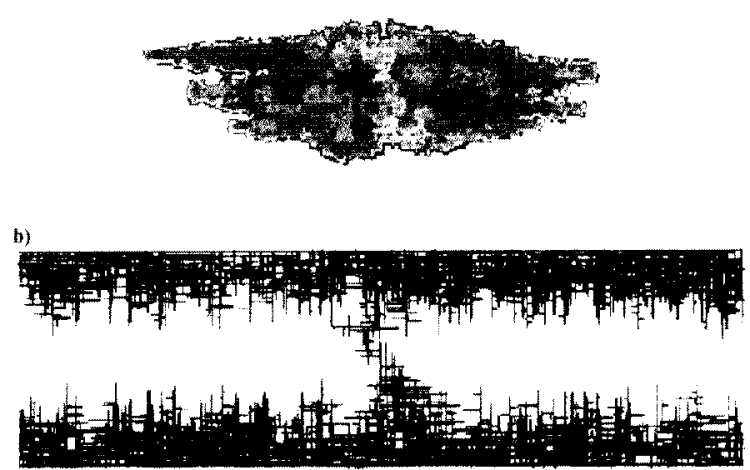

FIGURE 8 Cancellous and periosteal bone are being produced (a) after 23 cycles, with differentiation of mesenchymal cells caused by blood vessels $(b)$ reaching the edge of the cartilaginous model

of ossification is spreading out from the centre as the blood vessels start to invade the extremities of the bone. Considerable osteoblastic activity is still in evidence, especially at the ends of the bone where the formation of the metaphyses can be seen taking place. Most of the perichondrium of the original model can now be seen to have differentiated to periosteum. The blood vessel network (Figure 10b) now covers most of the matrix and ossification of the model is taking place at all points, noting that the blood vessels are now predominantly stable as the network ages.

After 50 cycles, the simulation has reached stage 10 where the growth of the model has stopped and any remaining growth zone chondrocytes are converted into normal chondrocytes, shown in Figure 11a. Ossification has now clearly spread to both ends of the bone and there are only a few regions of cartilage remaining. Many of the trabeculae have enclosed osteoblasts which have formed into osteocytes. Some osteoblastic activity can still be seen in areas of calcified cartilage. The model now continues to develop until all of the chondrocytes have matured. The network of blood vessels (Figure 11b) has finished growing at this point and covers the whole of the matrix. 

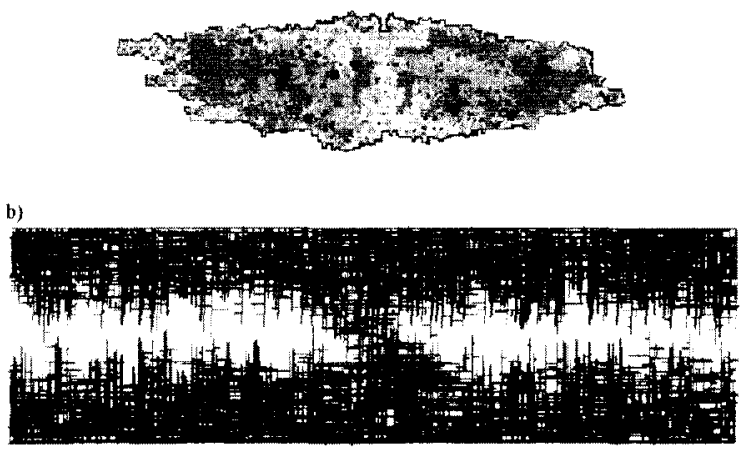

FIGURE 9 After approximately 30 cycles, a significant number of osteoblasts have been generated (a) due to the vascular invasion of the model (b). Most of these osteoblasts however have differentiated or died, but those along the sides of the model are still visible

a)
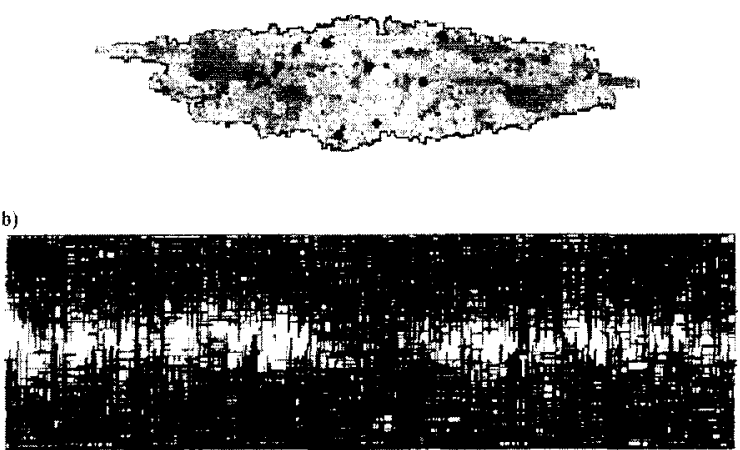

FIGURE 10 The formation of trabeculae may be observed (a) after 34 cycles, and most of the original perichondrium has differentiated into periosteum. The blood vessel network (b) now covers most of the matrix, noting that the blood vessels are now predominantly stable as the network ages

The end of the osteogenesis simulation run is shown in Figure 12a, having run for 103 cycles. All chondrocytes have matured, and all the cartilage has been converted into bone. It is notable that there is limited connectivity between the trabeculae, especially sorne of those towards the edges, and some of the trabeculae have no obvious support function. However, the structure produced appears visually a)

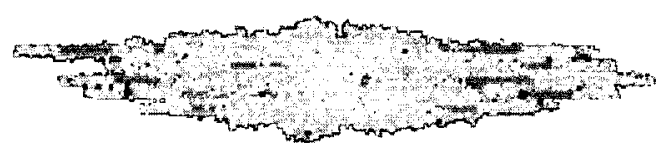

b)

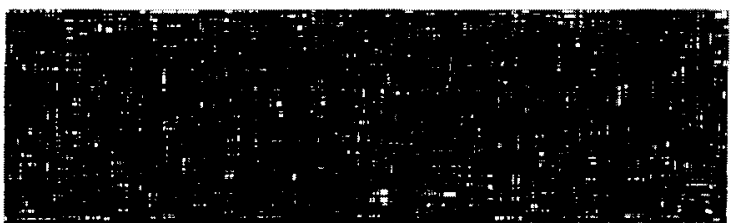

FIGURE 11 Growth has stopped after 50 cycles and the model (a) continues to develop until all of the chondrocytes have matured. Many of the trabeculae have enclosed osteoblasts which have formed into osteocytes. The network of blood vessels (b) has also finished growing and covers the whole of the matrix

comparable to a real section of cancellous bone from the vertebrae or calcaneus. The model is almost completely surrounded by a periosteum, and the larger trabeculae contain numerous osteocytes. Some osteogenic cells are also still in evidence. As expected, the corresponding blood vessel structure (Figure 12b) effectively shows no change from the previous stage.

a)

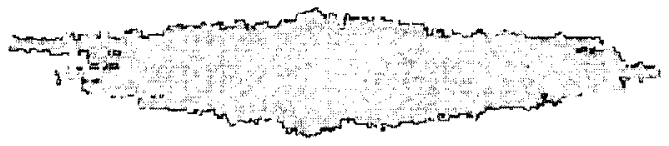

b)

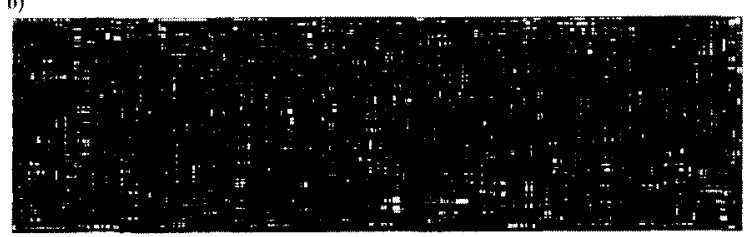

FIGURE 12 This typical osteogenesis simulation ran for 103 cycles, where (a) all chondrocytes have matured and all the cartilage has been converted into bone. As expected, the corresponding blood vessel structure (b) effectively shows no change from the previous stage 
The degree of variability due to the inherent stochastic nature of the simulation was investigated by running it to completion four times using the default settings, the final ages being 123, 104, 101 and 103 cycles respectively. The resulting bones, illustrated in Figure 13, demonstrate both interesting similarities and differences. In all four cases the blood vessel structure is very dense and made up entirely of stable vessels as would be expected. Further, the cartilage originally present has almost all been converted into bone, with only a small remnant left in the centre of some of the thicker trabeculae, and all of the bones have osteocytes embedded in them. It is also interesting to note that the bones are all more dense in the centre, due to the earlier vascular invasion that takes later here, and suggests that the initial matrix might be profitably changed to provide a more gentle gradient of vascular expansion. The bones do, however, show significant differences. The trabeculae are of different number and are in different places, and the external shape of the bones varies to a small degree, although the size is approximately constant. Although the basic structure of all the bones produced is the same, the internal details vary slightly. This is in concordance with what happens in real bone development and demonstrates the expected chaotic nature of the simulation.

The simulation was further evaluated by considering the effect of independently modifying several of the parameters, recording the final model structure. The end of growth age parameter (growth_maxage) was reduced from a default value of 50 to a new setting of 25 , the simulation ran for 85 cycles, producing the structure shown in Figure 14. The size of the bone is smaller since it has had less time to grow. Other than this, the structure produced has a similar shape and trabecular network to a bone produced with the default settings. Note that a little cartilage and calcified cartilage remains in the centres of some of the thicker trabeculae - this is normal and also happens in reality. The bone is well formed and almost completely covered by a periosteum. It is also interesting that although the program stopped growing after 25 cycles (all growth zone chondrocytes converted into ordinary chondrocytes and the parameters relating to
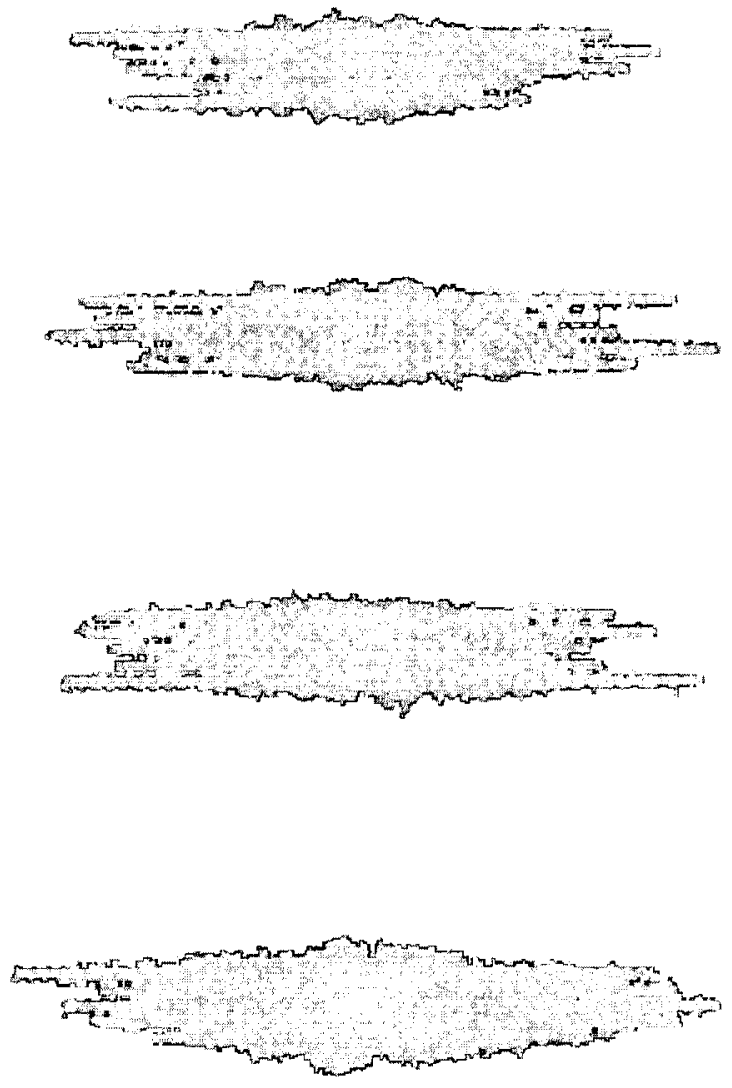

FIGURE 13 Final cell and tissue structures for four repeat runs using default settings. In all cases, the cartilage originally present has almost all been converted into bone, all of the bones have osteocytes embedded in them, and the bones are all more dense in the centre due to the earlier vascular invasion. The structures also demonstrate significant differences, the trabeculae are of different number and are in different places, and the external shape of the bones vary to a small degree, although the size is approximately constant

growth set to zero), the program continued for another 60 cycles. This is a similar number of extra cycles to that generally required for the 50 cycle program, suggesting that the process of the chondrocytes maturing etc. takes a relatively constant period of time, as would be expected. It should also be noted that, provided a sufficiently powerful computer is available, the model can grow much larger than has been demonstrated here. If this is required, it would be necessary to set growth_maxage to a larger value to allow sufficient time for the desired growth as well as changing the initial matrices. 


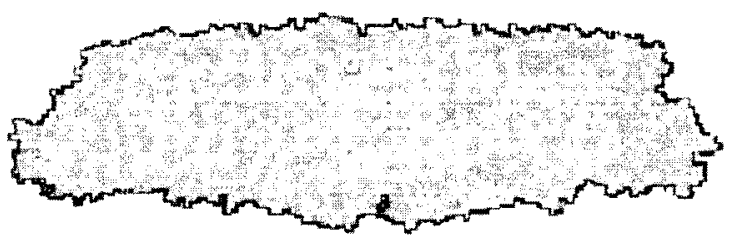

FIGURE 14 The effect of reducing the growth age parameter (growth_maxage) from a default value of 50 to a new setting of 25 , the size of the bone is smaller since it has had less time to grow, with other features consistent with the default setting

The osteoblast deposition constant (excluding calcified cartilage) parameter (ob_deposit2) was adjusted from the default value of 0.0005 to a new setting of 0.01 , the simulation running for 107 cycles and the resulting bone illustrated in Figure 15. Due to the higher value incorporated for this parameter, the simulation has deposited much more bone in marrow areas surrounding the calcified cartilage, and as a result, a much denser and more connected network of trabeculae is formed. The network looks much more like a set of holes in a solid block than the typical open networks produced by the program in default operation. One point to note is the increased prevalence of osteocytes (dark green). Since the osteoblasts were more likely to lay down bone in the marrow spaces, they were more likely to surround themselves in bone and therefore differentiate into osteocytes. Other than this, the bone looks similar to the default condition, the trabeculae run parallel to the length of the bone produced, with the spaces in between filled with marrow, along with a lining of periosteum on the outside. The simulation ran to a typical age and produced a bone of a typical shape.

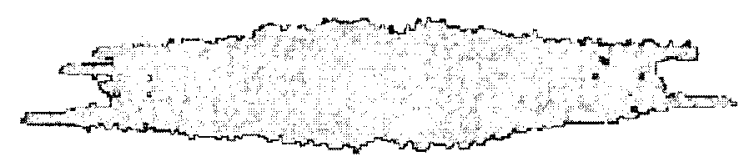

FIGURE 15 The effect of increasing the osteoblast deposition constant parameter (ob_deposit2) from the default value of 0.0005 to of 0.01 , the simulation has deposited much more bone in marrow areas surrounding the calcified cartilage, and as a result, a much denser and more connected network of trabeculae is formed
The osteogenic division factor (bv_choose) was modified from the default value of 0.9 to 0.5 , the simulation running for 104 cycles, illustrated in Figure 16. The lower value for this parameter has resulted in a greater proportion of osteoclasts being produced than would normally be the case. This leads to more resorption of the calcified cartilage before the bone is deposited on it, and also more resorption of the bone after it has been deposited. The greater resorption results in few trabeculae of appreciable size being formed with an associated lack of connectivity, and larger quantities of marrow being formed than observed using the default settings. Much of the bone that remains is in the form of small isolated fragments, not linked in a trabecular structure. The shape and size of the bone along with the final age however, are largely unaffected by this modification, and are typical for the simulation.

The hypertrophied chondrocyte growth constant (hcc_size) was increased from a default value of 0.5 to 1.0 , the simulation running for 133 cycles, illustrated in Figure 17. It was observed that all the chondrocytes mature to produce large hypertrophied chondrocytes, and therefore there are more larger holes in the lattice than would otherwise be expected. This again leads to a very open network of small, isolated fragments of bone since less of the original cartilage is left, due to the greater size of the hypertrophic chondrocytes. It is interesting to note that in the centre of the model, where vascular invasion takes place more quickly than elsewhere, the lattice is more connected. This is explained by the calcified cartilage having had less time to decay before it has become ossified by the action of osteoblasts, which in this case would be vascular in origin. Again the parameter adjustment makes little difference to the shape and size of the bone formed. The late age observed is probably due to the larger chondrocytes produced taking longer to completely die off and therefore delaying the termination of the program.

The maximum blood vessel growth distance (bv_grow_distance) was decreased from the default value of 4 to 1 , the simulation running for 120 cycles, illustrated in Figure 18a. The rate at which the blood 


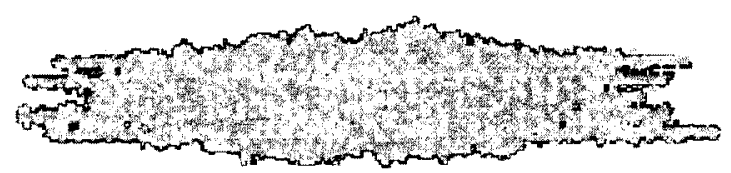

FIGURE 16 Reducing the osteogenic division factor (bv_choose) from 0.9 to 0.5 , has resulted in a greater proportion of osteoclasts being produced than would normally be the case, and hence few trabeculae of appreciable size being formed with an associated lack of connectivity

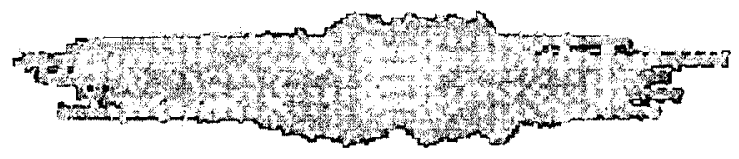

FIGURE 17 The effect of increasing the hypertrophied chondrocyte growth constant (hcc_size) from 0.5 to 1.0 , the chondrocytes mature to produce large hypertrophied chondrocytes and hence create larger holes in the lattice, again leading to a very open network of small, isolated fragments of bone

vessels grow has been greatly slowed down, leading to incomplete vascular invasion (Figure 18b) of the cartilagenous model and thus much of the calcified cartilage remains un-ossified. There are also mesenchymal cells remaining that have not differentiated for the same reason. As there is vascular development towards the centre of the model, this region has ossified more completely, leaving less calcified cartilage. It can also be seen that the calcified cartilage (light grey) has decayed, if ossification had taken place, quite an open structure would have formed.

\section{DISCUSSION}

The aim of this work was to develop an accurate and reliable stochastic simulation of cartilage bone osteogenesis. A thorough review of the literature was undertaken enabling cell types and tissues to be identified and a set of simulation rules to be established. The operation of the simulation has been evaluated longitudinally, reporting bone and blood vessel structures as the simulation develops to completion with

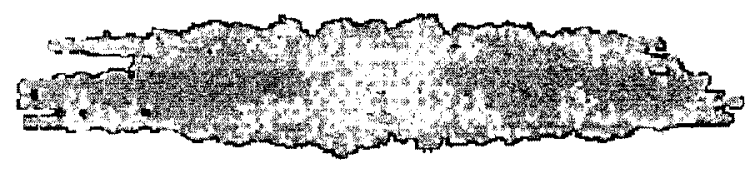

b)

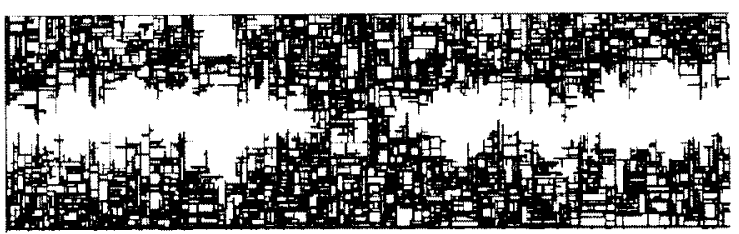

FIGURE 18 By decreasing the maximum blood vessel growth distance (bv_grow_distance) from 4 to 1 , the rate at which they grow has been reduced leading to incomplete vascular invasion of the cartilagenous model (b) and thus much of the calcified cartilage remains un-ossified (a)

fusion of the epiphyses; the simulation variability has been assessed by repeat runs using the same default conditions; and the effect of independently modifying key simulation parameters has been studied. The authors are not aware of a previously reported stochastic simulation of osteogenesis.

With reference to Table $\mathrm{V}$, there are two reasons for the surprisingly small value for the end of growth parameter (growth_maxage). Firstly, in real bone, the rate of growth slows down greatly after early infancy, then accelerates again in early adolescence, stabilising after approximately $18-20$ years. This is not currently modelled within the simulation, which has a constant rate of growth. Secondly, the matrix is set to a maximum size and the value of the end of growth parameter is set so that it does not exceed this size.

The authors acknowledge that osteoblasts lay down osteoid which is subsequently mineralised. However, for the purpose of this simulation, only the term 'bone' has been incorporated. Further development would be warranted for example if finite element analysis were to be implemented, since osteoid and mineralised bone have different mechanical properties.

The next stage for the development of this stochastic simulation of cartilage bone osteogenesis will be 
to demonstrate its utility, with potential avenues being paediatric clinical scenarios and therapeutic intervention.

The current limitations of this stochastic simulation of osteogenesis are primarily that there is minimal control of the overall shape of the bone being formed and the outer surface is too irregular; unrequired trabeculae are not removed (resorbed); diaphyseal wall growth is not incorporated, there is a lack of co-ordination between hypertrophication and periosteal ossification; and the simulation is $2 \mathrm{D}$ rather than 3D. Addressing these with reference to potential future developments of the simulation, it has recently been proposed (Langton et al, 1999) that the implementation of strain-weighting to a stochastic simulation of remodelling would remove isolated trabeculae and reduce surface irregularity by equilibriating the strain energy density experienced. The simulation primarily describes the development of cancellous rather than cortical bone and hence does not consider diaphyseal wall growth, and is therefore appropriate for simulation of irregular cartilage bones such as the vertebrae and calcanei rather than long bones. To improve the co-ordination between hypertrophication and periosteal ossification, initially it would be possible to change the initial matrix for the blood vessels to make them grow in a manner that causes them to reach any point on the surface when hypertrophication is taking place there. In the longer term, perhaps angiogenesis should be stimulated in a particular area of the bone by the presence of hypertrophied chondrocytes, as seems to be the case in nature. Development of a 3D simulation of osteogenesis will be a natural progression of the current $2 \mathrm{D}$ model.

In conclusion, a stochastic simulation of cartilage bone growth and formation has been developed and evaluated. The structures developed accurately follow the growth and form of irregular cartilage bones such as the vertebrae and calcanei. The future of the simulation is primarily dependent upon its potential utility in the field of bone metabolism and disease.

\section{References}

Baron, R. E. Anatomy and Ultrastructure of Bone. In: Favus, M. J. Ed. Primer on the Metabolic Bone Diseases and Disorders of
Mineral Metabolism, $3^{\text {rd }}$ edition. Philadelphia: Lippincott Raven; 1996; 3-10.

Carter, D. R., van der Meulen, M. C. H., and Beaupré, G. S. Skeletal Development. In: Marcus, R., Feldman, D. and Kelsey, J. Eds. Osteoporosis. San Diego: Academic Press Inc.; 1996; $333-350$.

Einhorn, T. A. The Bone Organ System: Form and Function. In: Marcus, R., Feldman, D. and Kelsey, J. Eds. Osteoporosis. San Diego: Academic Press Inc.; 1996; 3-22.

Gardner, E. Osteogenesis in the human embryo and fetus. In: The Biochemistry and Physiology of Bone. New York: Academic Press Inc.; 1956; 359-399.

Ham, A. W. and Leeson, T. S. Histology, 4th edition. London: Pitman Medical Publishing Co. Ltd.; 1961.

Hancox, N. The osteoclast. In: Bourne, G. H. Ed. The Biochemistry and Physiology of Bone. New York: Academic Press Inc.; 1956.

Jones, S. J., Glorieux, F. H., Travers, R., and Boyde, A. The Microscopic Structure of Bone in Normal Children and Patients with Osteogenesis Imperfecta: A Survey Using Backscattered Electron Imaging; from Calcified Tissue International; 64: 8-17; 1999.

Leblond, C. P. and Greulich, R. C. Autoradiographic studies of bone formation and growth. In: Bourne, G. H. The Biochemistry and Physiology of Bone. New York: Academic Press Inc.; 1956.

Moore, K. L. The Developing Human, $2^{\text {nd }}$ edition. Philadelphia: W.B. Saunders Co,; 1977.

Mosekilde, L. Consequences of the remodelling process for vertebral trabecular bone structure: a scanning electron microscopy study (uncoupling of loaded structures). Bone and Mineral 10: $13-35 ; 1990$.

Parfitt, A. M. Skeletal Heterogeneity and the Purposes of Bone Remodeling. In: Marcus, R., Feldman, D. and Kelsey, J. Eds. Osteoporosis. San Diego: Academic Press Inc.; 1996; 315329.

Pritchard, J. J. General anatomy and histology of bone. In: Boume G. H. Ed. The Biochemistry and Physiology of Bone. New York: Academic Press Inc.; 1956; 1-25.

Pritchard, J.J. The osteoblast. In: Bourne G. H. Ed. The Biochemistry and Physiology of Bone. New York: Academic Press Inc.; 1956; 179-211.

Shapiro, J. R. Osteogenesis Imperfecta and Other Defects of Bone Development as Occasional Causes of Adult Osteoporosis. In: Marcus, R., Feldman, D. and Kelsey, J. Eds. Osteoporosis. San Diego: Academic Press Inc.; 1996; 899-924.

Sinclair, D. An Introduction to Functional Anatomy, 5th edition. Oxford: Blackwell Scientific Publications; 1975; 28-38.

Sissons, H. A. The growth of bone. In: Bourne G. H. Ed. The Biochemistry and Physiology of Bone. New York: Academic Press Inc.; 1956; 443-474.

Thompson, D. W. On Growth and Form. Bonner, J. Y. Abridged edition Ed. Cambridge: University Press; 1971.

\section{References}

1. Haire, T. J., Hodgskinson, R., Ganney, P. S., and Langton, C. M. A comparison of porosity, fabric and fractal dimension as predictors of the Young's modulus of equine cancellous bone. Medical Engineering \& Physics 20: 588-593; 1998.

2. Langton, C. M., Haire, T. J., Ganney, P. S., Dobson, C. A., and Fagan, M. J. A dynamic stochastic simulation of the mechanical properties of cancellous bone associated with osteoporosis. Bone 22: 375-380; 1998. 
3. Langton, C. M., Haire, T. J., Ganney, P. S., Dobson, C. A., Fagan, M. J., Sisias, G., and Phillips, R. Stochastically simulated assessment of anabolic treatment following varying degrees of cancellous bone resorption. Submitted to Bone.

4. Percy, C. R. and Langton, C. M. Development of a fractal cancellous bone structure simulator. Submitted to Calcified Tissue International.
5. Rho, J. Y., Flaitz, D., Swarnakar, V., and Acharya, R. S. The characterization of broadband ultrasound attenuation and fractal analysis by biomechanical properties. Bone 20: 497 504; 1997.

6. Wolff, J. Das Gesetz der Transformation der Knochen. Hirschwald, Berlin; 1892. 


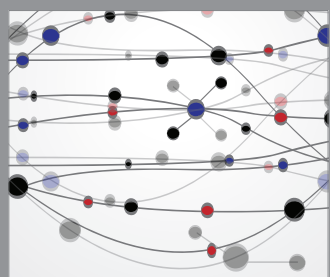

The Scientific World Journal
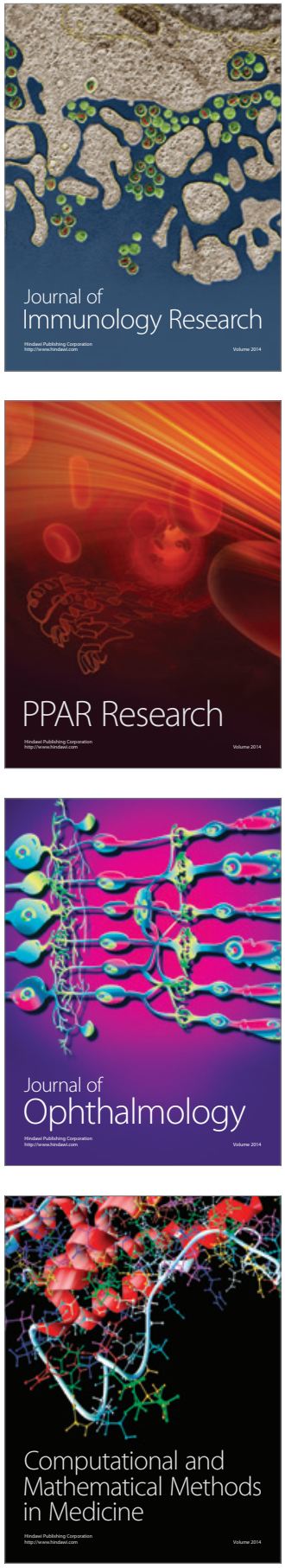

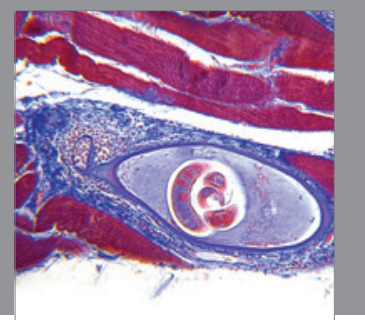

Gastroenterology

Research and Practice
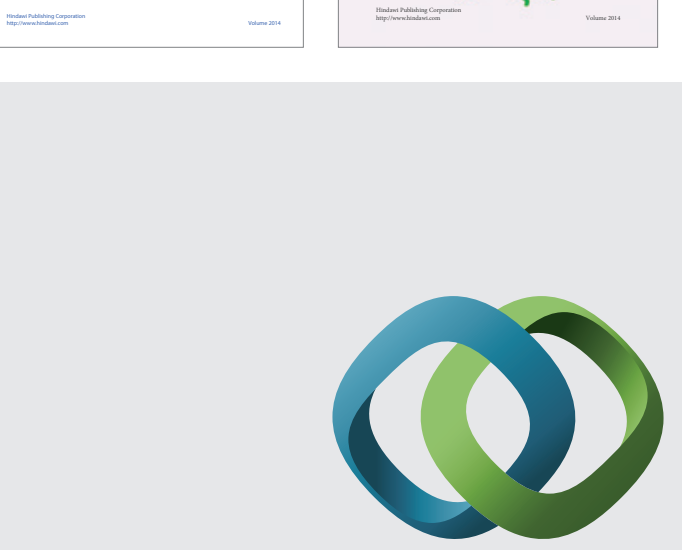

\section{Hindawi}

Submit your manuscripts at

http://www.hindawi.com
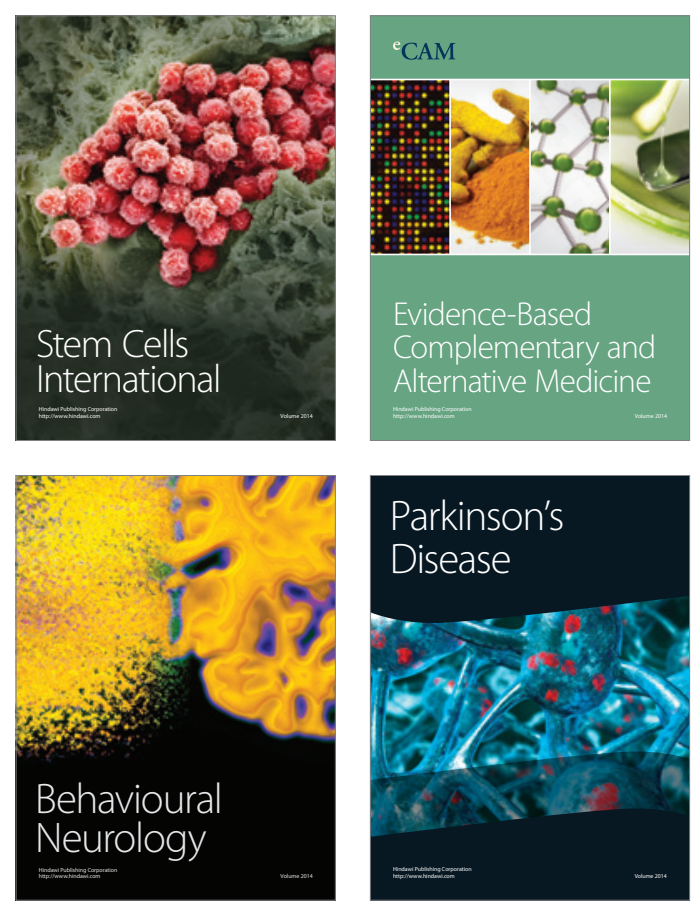

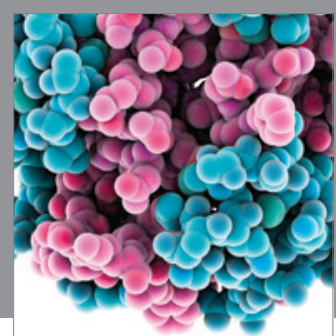

Journal of
Diabetes Research

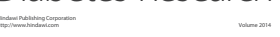

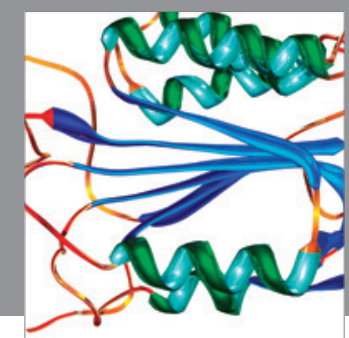

Disease Markers
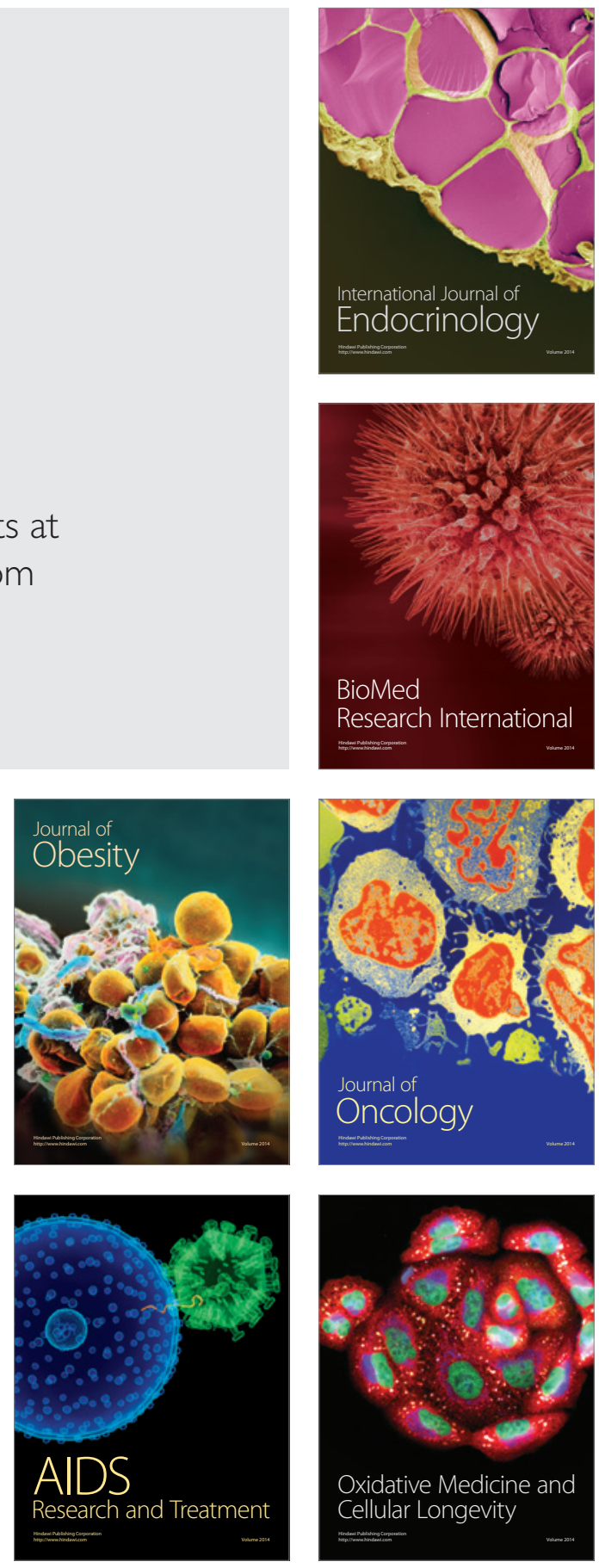